\title{
Safety and efficacy of oral triclofos in the evaluation of young and uncooperative children in pediatric ophthalmology clinic
}

\begin{abstract}
Aim: Evaluation of safety and efficacy of oral triclofos for eye examination of children under sedation.

Subjects and methods: This interventional prospective cohort study included children between 1 and 16 years of age. Children without an acute medical condition and normal cardio respiratory status, uncooperative for the relevant ocular examination were administered oral triclofos at a dose of $75 \mathrm{mg} / \mathrm{kg}$. The pulse oximetry, onset and duration of sedation, post sedation neurobehaviour and cognition were recorded.

Results: Thirty nine examinations under sedations (EUS) on thirty six consecutive children were performed. Of 36 children, 16 were females. The mean age was 2.9 year and the mean weight was $11.7 \mathrm{~kg}$. The mean duration of the onset of sedation was 64.7 minutes and the mean duration was 64.4 minutes. Of The drug did not have any effect on the pulse oximetry $(\mathrm{p}=0.68, \mathrm{p}=0.37)$. The correlation between the onset and duration of sedation with age and weight of the children was poor $(\mathrm{r}=0, \mathrm{r}=-0.1$ respectively). The boys slept earlier by mean 12 minutes $(p<0.01)$ than the girls $(\mathrm{p}<0.01)$ who slept for a mean duration of 16 minutes. A comprehensive eye examination could be completed in all the patients. The post sedation behavior and cognition remained normal in every child.

Conclusion: Oral triclofos at a dose of $75 \mathrm{mg} / \mathrm{kg} / \mathrm{dose}$ was safe and effective for examination of uncooperative children in pediatric ophthalmology practice. Further studies are needed to evaluate its effect on intraocular pressure measurement, on neurodevelopment and cognition and of the children.
\end{abstract}

Keywords: examination under anesthesia, examination under sedation, triclofos, oral sedation, eye examination in children
Volume 5 Issue 3 - 2016

\author{
Mihir Kothari, ${ }^{1,2}$ Kruti Shah,' Renu Singhania,' \\ Khushboo Shikhangi,' Swati Chavan' \\ 'Department of clinical outpatient pediatric ophthalmology, \\ Jyotirmay Eye Clinic and Ocular Motility Laboratory, India \\ ${ }^{2}$ Department of pediatric ophthalmology, Mahatme Eye Hospital, \\ India
}

Correspondence: Mihir Kothari, Jyotirmay eye clinic and ocular motility laboratory, 104/105 Kaalika Tower, Opp. Pratap Tower, Kolbad Road, Thane west 40060I, Maharashtra, India, Emaildrmihirkothari@jyotirmay.com

Received: October 27, 2016 | Published: November 28, 2016

\section{Introduction}

Evaluation of children under sedation or under anesthesia is often required in pediatric ophthalmology practice. Young children with developmental glaucoma, cataract, chronic uveitis, retinoblastoma, cerebral vision impairment etc. require such examinations repeatedly. However, there have been growing concerns regarding the long-term side-effects of single or repeated administration of anesthetic agents on the cognition, memory and behavior of the children. ${ }^{1-4}$ In a recent metaanalysis on inhalational pediatric anesthesia and neurodevelopmental impairments, the authors found a modestly elevated risk of adverse behavioral or developmental outcomes in children who were exposed to anesthesia/surgery during early childhood. ${ }^{2}$ A population-based retrospective cohort study found a $60 \%$ increased risk of learning disorders following more than one anesthetic exposure. ${ }^{5}$ Almost all the anesthetics have significant effects on neurotransmitters e.g. inhalant anesthetics such as Sevoflurane are GABA-ergic, while virtually all of the anesthetics in use viz. propofol, benzodiazepines, narcotics, and ketamine affects the NMDA neurotransmitter that has an effect on neuroplasticity.

Besides, an examination under anesthesia requires an anesthesiologist, systemic investigations, general fitness evaluation, well equipped operating room, post anesthesia monitoring, recovery room and involvement of additional health care personnel. All of that leading to a significant increase in the financial burden of such examination. On the other hand, chloral hydrate which is in use since Liebig first introduced into pediatric dentistry practice in 1832 , is the oldest, safe and the best studied sedative -hypnotic. ${ }^{6,7}$ Triclofos, the pharmacologically active metabolite of chloral hydrate is the phosphate ester of trichloroethanol and it is more stomach (lesser gastric irritation) and it is better accepted orally among the children. Oral triclofos has been successfully used as a sedative for young children in doses of $75-100 \mathrm{mg} / \mathrm{kg}$. ${ }^{89}$ In this study we found examination under sedation with oral triclofos in the dose of $75 \mathrm{mg} / \mathrm{kg}$ to be effective, safe and simple approach to perform satisfactory ocular examinations of young or uncooperative children under sedation in a pediatric ophthalmology centre.

\section{Subjects and methods}

This interventional prospective cohort study was performed at a dedicated teaching tertiary pediatric eye care centre between 1st May, 2015 to 31 st Dec, 2015. We included children between 1 year and 16 years of age who were not co-operative for relevant ocular examination. Children with cardio-respiratory distress, liver disease or an acute medical condition were excluded. After the initial evaluation by a pediatric optometrist and a pediatric ophthalmologist, if the child 
was adjudged to require the sedation, the parents were informed about the procedure. A thorough medical and ocular clinical history was taken from the patient's parents or guardian and the cardio respiratory status of the patient was checked using a pulse oximeter (Oxywatch, Choicemmed, India). The weight of the child was recorded according to the standard weighing guidelines on a weighing scale. Since oral triclofos is not associated with vomiting and the fasting in young children can be difficult, it was decided that the fasting was not needed for this study. However, once under sedation, the parents were asked to defer the administration of any food or liquids until the child had awakened.

Oral Triclofos (PediclorylTM, Dr. Reddy`s Laboratories Ltd., Hyderabad, India; $75 \mathrm{mg} / \mathrm{kg}$ ) was administered to the patients per oral by the hospital staff. The time of onset of sedation was recorded. The patients were under the direct observation of a nursing staff and a CCTV (closed circuit television) watch of the doctor. As soon as the child was asleep, he/she was moved to the examination room. The pulse oximetry was performed again and the values were recorded. The ophthalmic examinations by the pediatric optometrist and ophthalmologist were completed. In case, a child was found to be easily arousable during the transfer to the doctor's examination room or while being examined, the examination was withheld for a few seconds to a couple of minutes. In some patients the examination was withheld due to an active Bell's phenomenon (indicating lighter sedation) until the Bell's phenomenon was satisfactorily reduced. Adequate sedation and completion of the examination was defined as the depth of sedation when the comprehensive examination including the slit lamp biomicroscopy, retinoscopy, and indirect ophthalmoscopy, measurement of corneal diameter in glaucoma and measurement of intraocular pressure in all patients under sedation could be completed.

After completion of the examination, the parents were allowed to take the child home while in the sedated state. The administration of oral food/liquids was restricted until the child was fully awake. The parents were advised to report to the clinic if they noticed any physical/respiratory distress and also maintain the record of when the child recovered from the sleep. After a period of 48 hours, a follow up call was made by the clinic staff. The information about the duration of the sleep, post sedation activities (behavior and cognition), any sleep disturbances or any changes in the behavior and bowel habits were asked for. The data was entered in the excel sheet. For all the continuous, dependent variables (pre and post sedation pulse oximetry, onset and duration of sleep etc) a two tailed paired t test was used. (i.e.) and chi-square test was used for all the categorical and independent variables as a test of significance. $\mathrm{P}<0.05$ was considered statistically significant.

\section{Results}

Thirty nine examinations under sedations (EUS) were undertaken for thirty six consecutive children ( 3 children needed EUS twice). Of 36 children, 16 were females. The mean age was 2.9 year (standard deviation was \pm 1.6 , range was 1 -9years). The mean weight of the children was $11.7 \mathrm{~kg}( \pm 2.9,7.5-20 \mathrm{~kg})$. EUS was needed in eleven patients with strabismus, 10 with glaucoma, 5 with cerebral vision impairment (CVI) and 12 children had other anomalies (corneal opacity, cicatricial retinopathy of prematurity, retinal dystrophy and cataract). Mean duration of the onset of adequate sedation was 64.7 minutes $( \pm 54,10-270$ minutes, (Table 1$)$. The mean duration of the sedation (time to completely awake state from the time when child had slept) was 64.4 minutes $( \pm 66.5,10-300$ minutes $)$. The mean pulse rate while child was asleep was 93 beats per minute and mean spO2 of $97 \%$ which was statistically no different from (two tailed paired $t$ test, $p=0.68$ and $p=0.37$ respectively) pre sedation mean pulse rate of 91 beats per minute and mean $\mathrm{spO} 2$ level of $95 \%$.

There was a poor correlation between the onset and duration of sedation with age and weight of the children. The boys slept earlier (mean 12 minutes early) and for a longer duration than the girls (by a mean 16 minutes). Both, boys and girls, recovered from sedation after a mean duration of 129 minutes after the administration of the drug $( \pm 79,25-360)$. The examination could be successfully completed in all the patients. In three patients, the examination was performed in the waiting room rather than the examination room as the depth of sedation was light and attempt to shift the child to the examination room was associated with partial arousal of the child. In 21 patients, application of proparacaine $\mathrm{HCl}$ eye drops caused the child to partially arouse for a moment followed by continuation of the sleep. In two patients, following the application of the proparacaine $\mathrm{HCl}$ drops, the pediatric ophthalmologist had to wait for a few 'extra' minutes to proceed with the measurement of intraocular pressure of each eye to complete the examination.

Table I Comparison of age, weight and gender with the onset of sedation and mean duration of sleep

\begin{tabular}{|c|c|c|c|c|c|}
\hline \multirow{2}{*}{$\begin{array}{l}\mathbf{N}=39 \\
\text { Age }\end{array}$} & \multirow{2}{*}{$\begin{array}{l}\text { Mean (SD) } \\
2.9(1.6)\end{array}$} & \multicolumn{2}{|c|}{$\begin{array}{l}\text { Mean onset of sedation in minutes (Pearson } \\
\text { r) }\end{array}$} & \multicolumn{2}{|c|}{ Mean duration of sedation in minutes (Pearson $\mathbf{r}$ ) } \\
\hline & & $65(r=0.15)$ & & $64(r=0)$ & \\
\hline Weight & II.7 (3) & $65(0.26)$ & & $64(-0.0855)$ & \\
\hline \multirow[t]{3}{*}{ Gender } & & Boys & Girls & Boys & Girls \\
\hline & & $(n=20)$ & $(n=19)$ & $(n=20)$ & $(n=19)$ \\
\hline & & 59 minutes & 7I minutes & 72 minutes & 56 minutes \\
\hline$P$ value & & $<0.01$ & & $<0.01$ & \\
\hline
\end{tabular}




\section{Discussion}

Chloral hydrate is a sedative-hypnotic agent used for procedural sedation, particularly in the pediatric population..$^{7-9}$ CNS depression occurs via its active metabolite trichloroethanol. The exact mechanism of action is not known but action may be similar to barbiturates. ${ }^{10}$ In the literature there is only one case report of triclofos toxicity that too in a young preterm. ${ }^{11}$ At a therapeutic dose of $75 \mathrm{mg} / \mathrm{kg}$, triclofos does not cause any cardio respiratory ${ }^{12}$ or neurological disturbance and hence it is found to be safe in children with congenital heart disease who require the sedation for an echo cardiography. ${ }^{13,14}$ Triclofos is also safely used for neuroimaging of neurologically compromised children $^{15,16}$ and electroencephalography. ${ }^{17-19}$ Although triclofos is efficacious as a pediatric sedative agent,${ }^{20}$ it may not be safe in children with active wheeze. ${ }^{21}$ Oral triclofosis reported to cause cardiac arrhythmia at toxic doses in children with accidental poisoning. ${ }^{22-25}$ In the present study, no significant change in the pulse rate or $\mathrm{spO}_{2}$ was detected after administration of oral triclofos.

We found that the onset of sedation with triclofos was highly variable ranging from 10 minutes to as long as 270 minutes. Such a variability is reported by the previous investigators also., ${ }^{9,13-20}$ However, the new finding in this study was that the boys slept earlier and for a longer duration than the girls, unlike the previous studies. This may be because, our clinic was equipped with lots of play activities that were boy centric such as dashing cars and rocking horses that made boys more playful who got tired earlier than the girls who would play with dolls and the kitchen sets. Studies are needed to investigate any other scientific basis for gender dependant differences in the effect of oral triclofos.

We have been using oral triclofos for more than 8 years managing nearly 18 sedations every week. On informal questioning, the parents who have their children sedated repeatedly in our clinics have reported that the children sleep earlier if they had slept late on the previous night and they were made to wake up earlier in the morning on the examination day. Some parents have reported that the onset of sedation was rapid if the sedation was given during the routine sleep hours of the child e.g. At home, some children routinely slept in the afternoon and their parents found that the child would sleep faster if the sedative was given during that time. Repeated sedations have not resulted in the altered timing of onset and or the duration of sedation among the children who had multiple administrations of the drug. These findings will need further scientific validation in future studies.

Since the application of proproparacaine $\mathrm{HCl}$ eye drops caused partial arousal of the child, it is best to perform intraocular pressure measurement under topical anesthesia at the end. Probably, it is the sensation of the wetness from the eye lashes that causes arousal rather than any stinging from the drop. It is best to use a small drop of proproparacaine $\mathrm{HCl}$ to avoid spill over or the activation of eyelash reflex. The eyelash reflex is used by anesthesiologist to judge the depth of anesthesia. ${ }^{26}$ It remains intact in all the patients examined under triclofos sedation signifying a lower plane of sedation. ${ }^{26,27}$ The examiner should carefully avoid touching the eyelashes during the examination. We believe oral triclofos does not affect the intraocular pressure measurements and hence the measurements obtained under triclofos sedations could be more realistic than obtained under anesthesia. Further studies are needed to validate this hypothesis.
The completion rate of oral sedation in this study was $100 \%$. None of the patients underwent any painful procedure during the examination except one child for whom a forced duction test was successfully performed for monocular elevation deficiency. However, we routinely perform ocular biometry using contact a scan and hand held keratometry in our clinic for the children with cataract using oral triclofos. Occasionally we have removed corneal sutures (with difficulty) and excised the burst chalazion or pyogenic granuloma (without a difficulty) under oral triclofos sedation and topical proproparacaine $\mathrm{HCl}$. Till date we have had only one patient where oral ticlofos has been a repeated failure at inducing sufficient sleep. The child had bilateral congenital cataract, microphthalmos and glaucoma and she did not suffer from any specific identify able syndrome.

\section{Summary}

Oral triclofos at a dose of $75 \mathrm{mg} / \mathrm{kg} / \mathrm{dose}$ was safe and effective for the examination of children under sedation in an urban pediatric ophthalmology practice. Further studies are needed to evaluate the effect of oral triclofos on intraocular pressure measurement in children and its long term effect on the cognition and the neurodevelopment of the children.

\section{Funding}

None.

\section{Acknowledgments}

None.

\section{Conflicts of interest}

The authors declare that there was no conflict of interest.

\section{References}

1. Good WV. Is anesthesia safe for young children? J AAPOS. 2014;18(6):519-520.

2. DiMaggio C, Sun LS, Ing $C$, et al. Pediatric anesthesia and neurodevelopmental impairments: a Bayesian meta-analysis. J Neurosurg Anesthesiol. 2012;24(4):376-381.

3. Flick RP, Katusic SK, Colligan RC, et al. Cognitive and behavioral outcomes after early exposure to anesthesia and surgery. Pediatrics. 2011;128(5):1053-1061.

4. Stratmann G, Lee J, Sall JW, et al. Effect of general anesthesia in infancy on long-term recognition memory in humans and rats. Neuropsychopharmacology. 2014;39(10):2275-2287.

5. Wilder RT, Flick RP, Sprung J, et al. Early exposure to anesthesia and learning disabilities in a population-based birth cohort. Anesthesiology. 2009;110(4):796-804.

6. Bhatnagar S, Das UM, Bhatnagar G. Comparison of oral midazolam with oral tramadol, triclofos and zolpidem in the sedation of pediatric dental patients: an in vivo study. J Indian Soc Pedod Prev Dent. 2012;30(2):109114.

7. Needleman HL, Joshi A, Griffith DG. Conscious sedation of pediatric dentalpatients using chloral hydrate, hydroxyzine, and nitrous oxide- a retrospectivestudy of 382 sedations. Pediatr Dent. 1995;17(7):424-431. 
8. Gozal D, Dregenger B, Levin PD, et al. A pediatric sedation/anesthesia programme with dedicated care by anesthesiologists and nurses for procedures outside the operating room. J Pediatr. 2004;145(1):47-52.

9. da Costa LR, da Costa PS, Lima AR. A randomized double-blinded trial of chloral hydrate with or without hydroxyzine versus placebo for pediatric dental sedation. Braz Dent J. 2007;18(4):334-340.

10.@term+@DOCNO+3276. Access on 9th February, 2016.

11. Shahar E, Shnaps Y, Frand M. Acute triclofos poisoning in a preterm infant. ClinPediatr (Phila). 1979;18(11):706-707.

12. Jackson EA, Rabbette PS, Dezateux C, et al. The effect of triclofos sodium sedation on respiratory rate, oxygen saturation, and heart rate in infants and young children. Pediatr Pulmonol. 1991;10:40-45.

13. Napoli KL, Ingall CG, Martin GR. Safety and efficacy of chloral hydrate sedation in children undergoing echocardiography. $J$ Pediatr. 1996;129:287-291.

14. Layangool T, Sangtawesin C, Kirawittaya T, et al. A comparisonof oral chloral hydrate and sublingual midazolam sedation for echocardiogram in children. J Med Assoc Thai. 2008;91(S3):S45-S52.

15. Dacher JN, Neuenschwander S, Monroc M, et al. Sedation with oral hydroxyzine and rectal chloral hydrate in pediatric MRI and CT. J Radiol. 1996;77(12):1189-1192.

16. Low E, O’Driscoll M, MacEneaney P, et al. Sedation with oral chloral hydrate in children undergoing MRI scanning. Ir Med J. 2008;101(3):80 82 .
17. Millichap JG. Electroencephalographic evaluation of triclofos sodium sedation in children. Am J Dis Child. 1972;124(4):526-527.

18. Bektas O, Arica B, Teber S, et al. Chloral hydrate and/or hydroxyzine for sedation in pediatric EEG recording. Brain Dev. 2014;36:130-136.

19. Loewy J, Hallan C, Friedman E, et al. Sleep/sedation in children undergoing EEG testing: a comparison of chloral hydrate and music therapy. Am J Electroneurodiagnostic Technol. 2006;46(4):343-355.

20. Rumm PD, Takao RT, Fox DJ, et al. Efficacy of sedation of children with chloral hydrate. South Med J. 1990;83(9):1040-1043.

21. Mallol J, Sly PD. Effect of chloral hydrate on arterial oxygen saturation in wheezy infants. Pediatr Pulmonol. 1988;5(2):96-99.

22. DiGiovanni AJ. Reversal of chloral hydrate-associated cardiac arrhythmia by a beta-adrenergic blocking agent. Anesthesiology. 1969;31(1):93-97.

23. Nordenberg A, Delisle G, Izukawa T. Cardiac arrhythmia in a child due to chloral hydrate ingestion. Pediatrics. 1971;47:134-135.

24. Gustafson A, Svensson SE, Ugander L. Cardiac arrhythmias in chloral hydrate poisoning. Acta Med Scand. 1977;201:227-230.

25. Wiseman HM, Hampel G. Cardiac arrhythmias due to chloral hydrate poisoning. Br Med J. 1978;2(6142):960.

26. Guedel AE. Inhalation anesthesia, $2^{\text {nd }}$ edition. USA: Macmillan; 1951.

27. Vuyk J, Engbers FH, Lemmens HJ, et al. Pharmacodynamics of propofol in female patients. Anesthesiology. 1992;77:3-9. 